\title{
Real-Time Quaking-Induced
} Conversion Detection of $\mathrm{PrPSc}^{\mathrm{Sc}}$ Fecal Samples From Chronic Wasting Disease Infected White-Tailed Deer Using Bank Vole Substrate

\section{OPEN ACCESS}

Edited by:

Fabrizio Passamonti,

University of Perugia, Italy

Reviewed by:

Akikazu Sakudo,

Okayama University of Science, Japan Peter Heegaard,

Technical University of

Denmark, Denmark

*Correspondence:

Eric M. Nicholson

eric.nicholson@ars.usda.gov

Specialty section:

This article was submitted to Veterinary Infectious Diseases,

a section of the journal

Frontiers in Veterinary Science

Received: 18 December 2020 Accepted: 03 February 2021

Published: 04 March 2021

Citation:

Hwang S, Greenlee JJ and Nicholson EM (2021) Real-Time

Quaking-Induced Conversion Detection of PrPSc in Fecal Samples

From Chronic Wasting Disease Infected White-Tailed Deer Using Bank

Vole Substrate.

Front. Vet. Sci. 8:643754. doi: 10.3389/fvets.2021.643754

\section{Soyoun Hwang, Justin J. Greenlee and Eric M. Nicholson*}

Virus and Prion Research Unit, National Animal Disease Center, United States Department of Agriculture, Agricultural Research Service, Ames, IA, United States

Chronic wasting disease (CWD) is a transmissible spongiform encephalopathy (TSE) that is fatal to free-range and captive cervids. CWD has been reported in the United States, Canada, South Korea, Norway, Finland, and Sweden, and the case numbers in both wild and farmed cervids are increasing rapidly. Studies indicate that lateral transmission of cervids likely occurs through the shedding of infectious prions in saliva, feces, urine, and blood into the environment. Therefore, the detection of CWD early in the incubation time is advantageous for disease management. In this study, we adapt realtime quacking-induced conversion (RT-QulC) assays to detect the seeding activity of CWD prions in feces samples from clinical and preclinical white-tailed deer. By optimizing reaction conditions for temperature as well as the salt and salt concentration, prion seeding activity from both clinical and preclinical animals were detected by RT-QuIC. More specifically, all fecal samples collected from 6 to 30 months post inoculation showed seeding activity under the conditions of study. The combination of a highly sensitive detection tool paired with a sample type that may be collected non-invasively allows a useful tool to support CWD surveillance in wild and captive cervids.

Keywords: CWD, prion disease, RT-QuIC, transmissible spongiform encephalopathy, TSE, feces, white-tailed deer

\section{INTRODUCTION}

Chronic wasting disease (CWD) is a form of transmissible spongiform encephalopathy (TSE) or prion disease affecting cervids including deer, elk, reindeer, and moose. Prion diseases result from the misfolding of the cellular prion protein $\left(\mathrm{PrP}^{\mathrm{C}}\right)$ into a pathogenic form $\left(\mathrm{PrP}^{\mathrm{Sc}}\right)$. Other prion diseases include scrapie in sheep, bovine spongiform encephalopathy (BSE) in cattle, and Creutzfeldt-Jakob disease (CJD), fatal familial insomnia (FFI), Gerstmann-Sträussler-Scheinker syndrome (GSS), and kuru in humans. CWD has been reported across North America including 26 states in the United States and three Canadian provinces. CWD-infected animals have also been reported in South Korea, Norway, Finland, and most recently, Sweden (1-4).

Chronic wasting disease infected cervids have misfolded prion proteins distributed widely, not only in the nervous system but also in lymphoid tissues, muscle, and blood (5-7). These animals are known to shed prions into the environment via saliva, urine, blood, and feces. This environmental contamination is often suggested to be the cause for horizontal CWD transmission among captive 
and free-ranging wild animals. Despite the awareness of potential sources, rising case numbers in wild cervids highlight the lack of effective CWD management strategies. Lacking treatment or vaccination, any management strategy for CWD will be dependent on sensitive and early detection of CWD prions in CWD infected animals. Early detection in a readily accessible sample that can be collected in a non-invasive procedure will afford producers and regulatory entities the opportunity to test prior to shipment as well as upon receipt to reduce the likelihood that an infected animal would be introduced into an otherwise healthy herd. Samples that are shed from animals, particularly early in the incubation period, have low concentrations of detectable prions, which necessitates highly sensitive prion detection methods. Highly sensitive prion detection tools like real-time quaking induced conversion (RT-QuIC) that amplify the prion in vitro enabled the detection of prions from early stage of the disease and from various sample types in addition to brain and lymphoid tissues. Several reports have indicated that fecal prions as well as those found in saliva, blood, and urine from cervids could be detected using RT-QuIC, in some cases using preclinical samples (8-14).

In the study, we tested the suitability of bank vole prion protein $(\mathrm{BV} \mathrm{rPrP}$ ) as a substrate for CWD detection in feces samples from clinical and preclinical white-tailed deer. To accomplish this, the reaction conditions were optimized using different enrichment methodologies, different salt concentrations, different temperatures, and different salt ions to amplify fecal prions for improved sensitivity and specificity.

\section{MATERIALS AND METHODS}

\section{Source of Fecal Samples}

All fecal samples used in this manuscript were collected from white-tailed deer that were experimentally inoculated by the oronasal route with the CWD agent from either a white-tailed deer (deer numbers \#1548 and \#1553) or an experimentally inoculated raccoon (deer numbers \#1542 and \#1555). Feces was collected from two non-inoculated, non-CWD exposed deer for use as negative controls (deer numbers \#831 and \#1801). They are summarized in Table 1. All animal experiments were

TABLE 1 | Animal experimental summary of genotype, inoculum, survival period, and enzyme-immunoassay (EIA) in white-tailed deer inoculated oronasally with the agent of chronic wasting disease from white-tailed deer and racoon.

\begin{tabular}{lclcl}
\hline Eartag & \begin{tabular}{c} 
Genotype \\
\cline { 2 - 4 }
\end{tabular} & CWD Inoculum & $\begin{array}{c}\text { Months post- } \\
\text { incubation (mo.) }\end{array}$ & b EIA O.D. \\
\hline 1553 & GG & WTD & 21.4 & 4.00 \\
1548 & GG & WTD & 24 & 4.00 \\
1542 & GG & Racoon CWD & 34.5 & 4.00 \\
1555 & GG & Racoon CWD & a 56.7 & NT \\
831 & GG & Neg. control & 42 & ${ }^{\circ} 0.07$ \\
1801 & GG & Neg. control & 34.1 & ${ }^{\circ} 0.08$ \\
\hline
\end{tabular}

NT indicates that samples were not tested.

a This animal has not developed clinical signs at the time of this study.

bIDEXX HerDCheck CWD Ag test.

${ }^{c}$ Values below 0.18 are negative. conducted at the National Animal Disease Center under the approval of the Institutional Animal Care and Use Committee (protocol number: ARS-2018-748, date of approval from ethical committee: August 7, 2015). The animal experiments were carried out in accordance with the Guide for the Care and Use of Laboratory Animal (Institute of Laboratory Animal Resources, National Academy of Sciences, Washington DC, USA). Each deer was inoculated oronasally similar to previously described (15) with $1 \mathrm{ml}$ of $10 \%$ (wt/vol) brain homogenate from a single animal with clinical signs consistent with prion disease and confirmed positive by immunohistochemistry, enzymeimmunoassay (EIA), and Western blot. Fecal samples were collected at $\sim 6$-month intervals until the deer developed clinical signs consistent with CWD. The mean incubation period was 682 days for deer inoculated with the CWD agent from a whitetailed deer. At $\sim 1,700 \mathrm{dpi}$, the experiment with deer inoculated with the CWD agent from raccoons is ongoing, but deer \#1542 was euthanized at 1,035 dpi due to clinical signs consistent with CWD.

\section{Preparation of Cervid Feces Extracts}

Cervid feces was prepared as described by Cheng et al. (9). Briefly, $1 \mathrm{~g}$ of previously collected fecal pellets was weighed and added into the feces extract buffer ( $20 \mathrm{mM}$ sodium phosphate ( $\mathrm{pH} 7.1$ ), $130 \mathrm{mM} \mathrm{NaCl}, 0.05 \%$ Tween $20,1 \mathrm{mM}$ phenylmethylsulfonyl fluoride (PMSF), and 1X complete protease inhibitors (Roche) giving a final concentration of $10 \%(\mathrm{w} / \mathrm{v})$. Then, the fecal pellets were homogenized in $M$ tubes (GentleMACS M tubes) using a dissociator (GentleMACS, Miltenyi Biotec) for a minute with two to three repeats to ensure complete pellet disruption. The tubes were then placed onto an orbital shaker for $1 \mathrm{~h}$ at room temperature. After centrifugation at $18,000 \times \mathrm{g}$ for $5 \mathrm{~min}$, supernatants were collected and stored at $-80^{\circ} \mathrm{C}$ for further use.

\section{Sodium Phosphotungstic Acid Precipitation}

To each $1 \mathrm{ml}$ volume of $10 \%$ (w/v) fecal supernatant, $250 \mu \mathrm{l}$ of $10 \% \mathrm{~N}$-lauryl sarcosine was added. Samples were then incubated at $37^{\circ} \mathrm{C}$ for $30 \mathrm{~min}$ at $1,400 \mathrm{rpm}$ in a thermomixer. Using a stock solution of $10 \%$ sodium phosphotungstic acid (NaPTA), $170 \mathrm{mM}$ of magnesium chloride ( $\mathrm{pH}$ 7.4) was added to each sample to give a final concentration of $0.3 \% \mathrm{NaPTA}$ in the samples. Samples were incubated at $37^{\circ} \mathrm{C}$ for $2 \mathrm{~h}$ with shaking at $400 \mathrm{rpm}$. Supernatants were removed following centrifugation at $15,800 \times \mathrm{g}$ for $30 \mathrm{~min}$. Pellets were washed with wash buffer [10 mM Tris-Cl, pH 7.5, $100 \mathrm{mM} \mathrm{NaCl,} \mathrm{0.5 \%} \mathrm{Triton-X} \mathrm{100,}$ $10 \mathrm{mM}$ ethylenediamine tetraacetic acid (EDTA), 0.5\% sodium deoxycholate $(\mathrm{w} / \mathrm{v})$, and $0.1 \%$ sarkosyl $(\mathrm{w} / \mathrm{v})]$, and centrifuged again for $15 \mathrm{~min}$ at 15,800 g. Pellets were resuspended in $100 \mu \mathrm{l}$ of Dulbecco's phosphate-buffered saline (DPBS) with $0.05 \%$ Sodium dodecyl sulfate (SDS).

\section{PAD-Beads Enrichment}

PAD-Beads and all buffers were provided as a kit from (Microsens, London, UK). Standard PAD-Beads enrichment was followed as described by Hwang et al. (16). Briefly, $200 \mu \mathrm{l}$ of $10 \%$ $(\mathrm{w} / \mathrm{v})$ fecal samples was mixed with $500 \mu \mathrm{l}$ of distilled water by gently tapping the tubes, and then $200 \mu \mathrm{l}$ of capture buffer and 
$100 \mu \mathrm{l}$ of beads were added to the tube. The tubes were then shaken for $30 \mathrm{~min}$ at room temperature on a rocker. After the incubation, beads were captured on a magnet and the liquid was removed. Then, the samples were washed with wash buffer 1 and wash buffer 2 . For elution, $25 \mu \mathrm{l}$ of elution buffer $(0.1 \mathrm{M} \mathrm{NaOH}$, $0.1 \%$ Triton X-100) was added to the beads, and the tubes were shaken for $5 \mathrm{~min}$. The tubes were placed on a magnet to capture the beads. While the tubes were on the magnet, the same volume, $25 \mu \mathrm{l}$ of $0.1 \mathrm{M} \mathrm{HCl}$ was added to the tubes to neutralize the $\mathrm{NaOH}$ and mixed gently. Finally, the liquid was removed and analyzed by RT-QuIC.

For large scale sample preparation, $1 \mathrm{ml}$ of fecal samples was used instead of $200 \mu \mathrm{l}$, and all other buffers were used at five times the previously indicated volume except the elution buffer and $\mathrm{HCl}$ neutralization which were both $50 \mu \mathrm{l}$.

\section{Recombinant Prion Protein Production and Purification}

Escherichia coli [BL21 ( $\lambda \mathrm{DE} 3)]$ was transformed with the pET28a vector containing the bank vole PrP gene (amino acids 23-231; GenBank accession number AF367624), and the recombinant bank vole prion proteins were expressed and purified as described by Vrentas et al. (17). The concentration of pooled protein eluent was measured by UV and calculated from the absorbance at $280 \mathrm{~nm}$ using an extinction coefficient of $62,005 \mathrm{M}^{-1} \mathrm{~cm}^{-1}$ as calculated for the bank vole prion protein (18).

\section{Real-Time Quaking-Induced Conversion Protocol}

Real-time quaking-induced conversion reactions were performed as previously described (19-25). The reaction mix was composed of $10 \mathrm{mM}$ phosphate buffer ( $\mathrm{pH} 7.0$ ), either 100, 200, 300, 400, and $500 \mathrm{mM} \mathrm{NaCl}$ or $100,200,300,400$, and $500 \mathrm{mM}$ sodium iodide $(\mathrm{NaI}), 0.1 \mathrm{mg} / \mathrm{ml}$ recombinant bank vole prion protein, $10 \mu \mathrm{M}$ thioflavin $\mathrm{T}$ (ThT), and $1 \mathrm{mM}$ EDTA tetrasodium salt. Aliquots of the reaction mix $(98 \mu \mathrm{L})$ were loaded into each well of a black 96-well plate with a clear bottom (Nunc, Thermo Fisher Scientific, USA) and seeded with $2 \mu \mathrm{L}$ of fecal homogenate dilutions with $0.05 \%$ SDS. The plate was then sealed with plate sealer film and incubated at 37,42 , or $48^{\circ} \mathrm{C}$ in a $\mathrm{BMG}$ FLUOstar Omega plate reader with cycles of 1 min shaking (700 rpm double orbital) and $1 \mathrm{~min}$ rest for $100 \mathrm{~h}$. ThT fluorescence measurements (excitation $460 \mathrm{~nm}$, emission $480 \mathrm{~nm}$, bottom read, 20 flashes per well, and manual gain 1,400) were taken every $45 \mathrm{~min}$.

All RT-QuIC assays for each dilution of each sample were performed as two repeats of four replicates for a total of eight replicates. ThT fluorescence data are displayed as the average ThT fluorescence of four technical replicates for each time point and, to be considered positive, the ThT fluorescence of at least two replicates out of four reactions must be positive. Positive threshold was calculated as the mean value of noninoculated control sheep brain homogenates plus 10 SDs, and lag time is defined as the time to reach the positive threshold $(20,26,27)$.

\section{RESULTS}

\section{Real-Time Quaking-Induced Conversion Detection of PrPSc in Non-enriched Fecal Samples From White-Tailed Deer Clinically Affected With CWD}

To test whether CWD prions from fecal samples, without enrichment or substrate replacement, could be detected by RT-QuIC using BV rPrP, reactions were seeded with different dilutions of fecal samples collected from clinically affected whitetailed deer at the time of necropsy. Different concentrations of $\mathrm{NaCl}$ were tested to find the optimal reaction conditions with $\mathrm{BV}$ rPrP and CWD infected deer fecal samples (Figure 1). All tested conditions showed an increase in ThT fluorescence typical for the detection of TSE within $30 \mathrm{~h}$ except $300 \mathrm{mM} \mathrm{NaCl}$. Using higher salt concentrations (400 and $500 \mathrm{mM} \mathrm{NaCl}$ ) improved the seeding activity with shorter lag time for assays seeded with feces from positive animal samples while assays seeded with feces from negative control samples remain below the threshold to be considered positive (Figures 1D,E).

\section{Real-Time Quaking-Induced Conversion Detection of PrPSc in Enriched Fecal Samples by NaPTA Precipitation From White-Tailed Deer Clinically Affected With CWD}

To evaluate the efficacy of NaPTA precipitation in detection CWD prions in fecal samples from clinical CWD infected whitetailed deer, reactions containing recombinant $\mathrm{BV}$ rPrP were seeded with different dilutions of NaPTA enriched fecal samples. Again, different concentrations of $\mathrm{NaCl}$ were tested to find the optimal condition for the detection of $\mathrm{PrP}^{\mathrm{Sc}}$ in the fecal samples with RT-QuIC following NaPTA precipitation. Similar to the results observed for non-enriched samples, all tested $\mathrm{NaCl}$ concentrations, except $300 \mathrm{mM} \mathrm{NaCl}$, result in fibril seeding based on an observed increase in ThT fluorescence (Figure 2). Assays with higher $\mathrm{NaCl}$ concentrations (400 and $500 \mathrm{mM}$ ) showed shorter lag times with spontaneous conversion with low intensity and extended lag time at $70 \mathrm{~h}$. It is clear that NaPTA precipitation improved the RT-QuIC detection in fecal samples by shortening lag time of seeding activity.

\section{Real-Time Quaking-Induced Conversion Detection of CWD Prions in Fecal Samples of White-Tailed Deer With Clinical Signs of CWD Following PAD-Bead Enrichment}

PAD-Beads, a commercially available kit, has been used to successfully enrich brain homogenate samples prior to RT-QuIC (16). To evaluate the efficacy of PAD-Beads enrichment for CWD prion detection from fecal samples, reactions containing $\mathrm{BV} \mathrm{rPrP}$ were seeded with different dilutions of PAD-Beads eluate in the presence of different $\mathrm{NaCl}$ concentrations. Most assays did not show any increase of ThT. However, assays containing $100 \mathrm{mM}$ $\mathrm{NaCl}$ showed the increase of ThT fluorescence for the assays seeded with fecal sample of animal \#1553 (Figure 3). Given the 

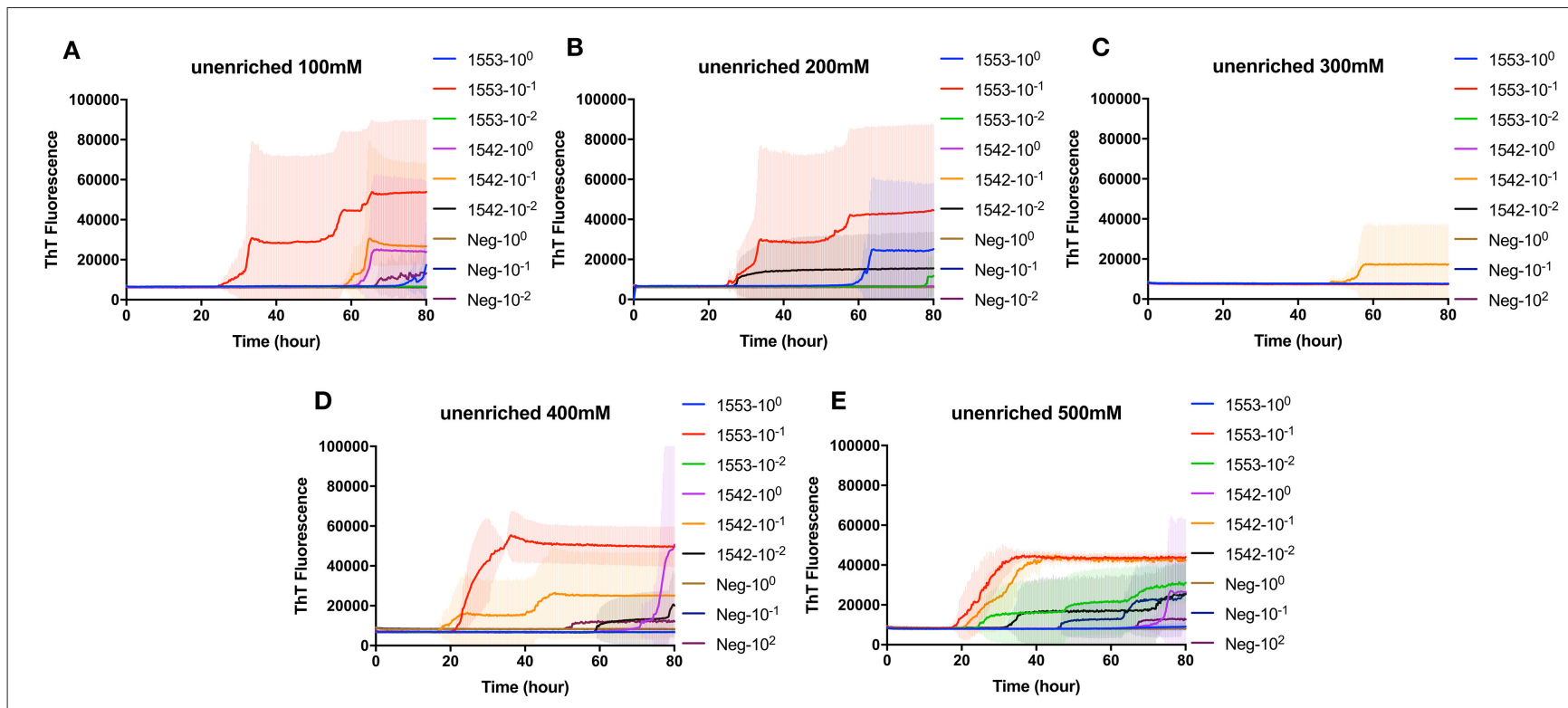

FIGURE 1 | Real-time quacking-induced conversion (RT-QulC) reactions containing different $\mathrm{NaCl}$ concentrations (A) $100 \mathrm{mM}$, (B) $200 \mathrm{mM}$, (C) $300 \mathrm{mM}$, (D) $400 \mathrm{mM}$, and (E) $500 \mathrm{mM}$ seeded with fecal dilutions from chronic wasting disease (CWD) infected white-tailed deer brains using BV rPrP as a substrate. RT-QulC reactions were seeded with $10^{0}, 10^{-1}$, or $10^{-2}$ dilutions of $10 \%$ fecal homogenate. All reactions were seeded with fecal homogenate of white-tailed deer with the addition of $0.001 \%$ of SDS. Shown are the average ThT fluorescence readings determined from all replicates (four replicate reactions per each dilution).

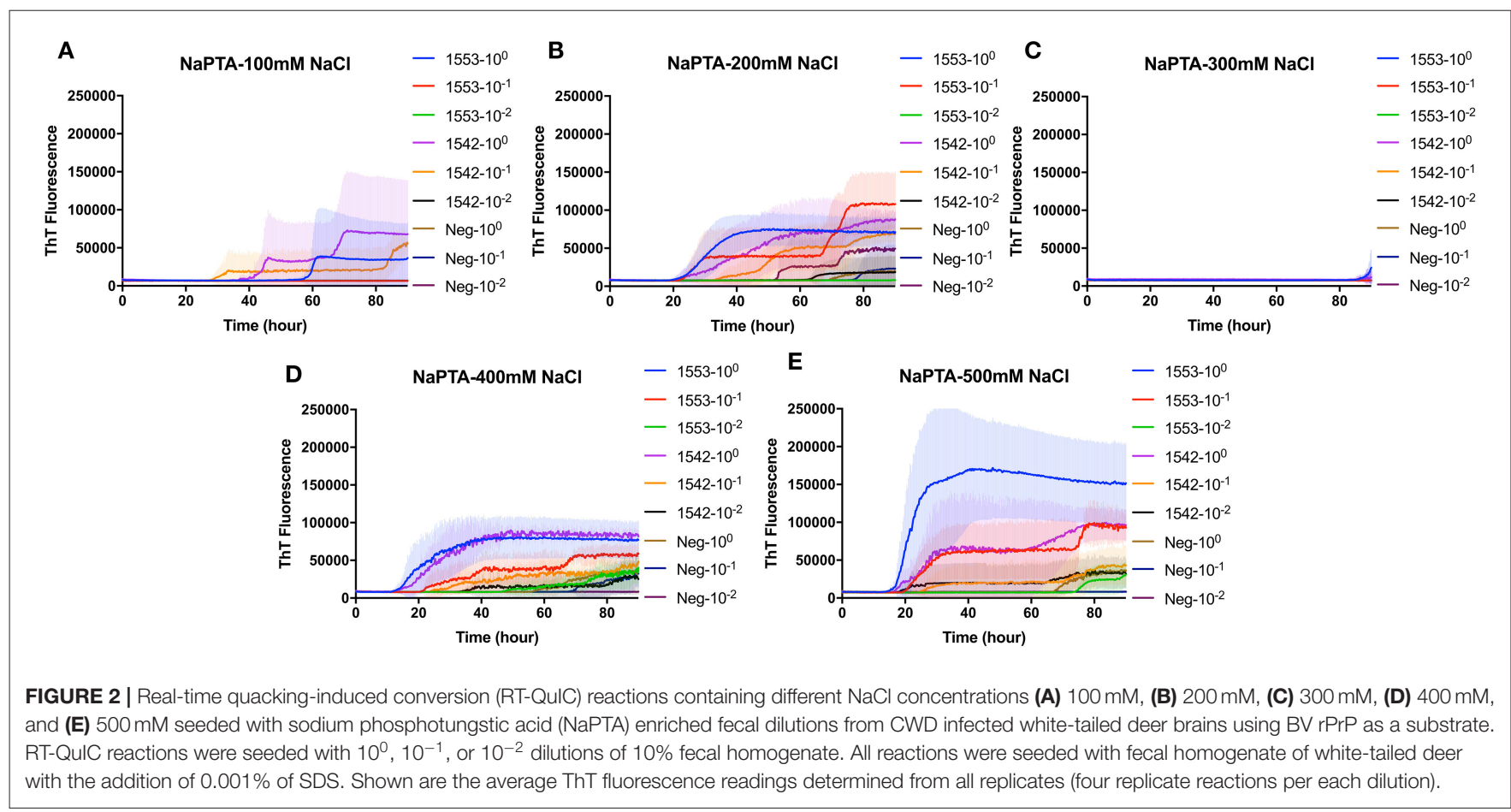

presumably lower concentration of $\mathrm{PrP}^{\mathrm{Sc}}$ in fecal samples relative to brain homogenate, we also assessed a higher starting volume of fecal sample homogenate. The standard PAD-Bead protocol uses $200 \mu \mathrm{l}$ of $10 \%$ fecal homogenates. Therefore, instead of using $200 \mu \mathrm{l}$ of $10 \%$ fecal homogenates (standard protocol), $1 \mathrm{ml}$ of $10 \%$ fecal homogenate was used for PAD-Beads enrichment.
All other reagents in the protocol were also increased by five times. At the end, $100 \mu \mathrm{l}$ of eluate was collected to make the final 1/10 volume of original fecal sample, a volume equivalent to that used in NaPTA enrichment. When assays were seeded with fecal eluate of large scale PAD-Beads enrichment, every assay showed rapid (within $20 \mathrm{~h}$ ) conversion. However, assays 


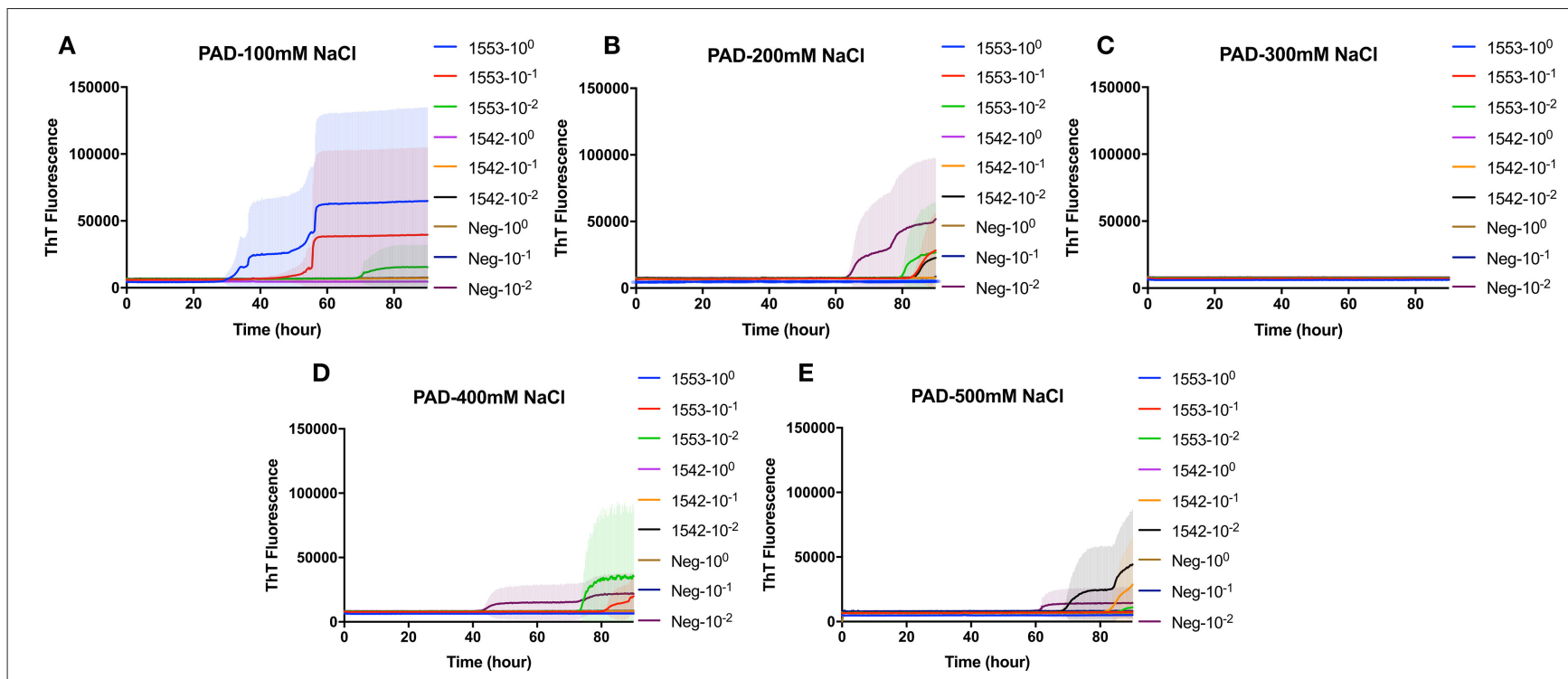

FIGURE 3 | Real-time quacking-induced conversion (RT-QulC) reactions containing different $\mathrm{NaCl}$ concentrations (A) $100 \mathrm{mM}$, (B) $200 \mathrm{mM}$, (C) $300 \mathrm{mM}$, (D) $400 \mathrm{mM}$, and (E) $500 \mathrm{mM}$ seeded with PAD-Beads enriched fecal dilutions from CWD infected white-tailed deer brains using BV rPrP as a substrate. RT-QulC reactions were seeded with $10^{0}, 10^{-1}$, or $10^{-2}$ dilutions of $10 \%$ fecal homogenate. All reactions were seeded with fecal homogenate of white-tailed deer with the addition of $0.001 \%$ of SDS. Shown are the average ThT fluorescence readings determined from all replicates (four replicate reactions per each dilution).

seeded the equivalently treated negative control fecal samples showed seeding activity with only a marginally longer lag time. Specifically, assays seeded with negative control in the presence of $400 \mathrm{mM} \mathrm{NaCl}$ had a lag time $\sim 5 \mathrm{~h}$ longer than from positive animals, and all reactions containing $500 \mathrm{mM} \mathrm{NaCl}$ showed the increase of ThT fluorescence in a similar lag time albeit lower intensity (Figures 4A,B). ThT fluorescence comparing reactions seeded with unenriched feces, enriched by large scale PAD-Beads, and NaPTA is shown in Figure 4. Based on these results, PADBead enrichment was not further pursued for fecal samples, and NaPTA enrichment was used for all further experiments.

\section{Real-Time Quaking-Induced Conversion Detection of CWD Prions in Fecal Samples Collected From White-Tailed Deer in the Preclinical Stage of Prion Disease}

To evaluate if RT-QuIC assays can detect prions from fecal samples of preclinical white-tailed deer, fecal samples that were collected at times of routine animal handling were also seeded in RT-QuIC reactions. In total, eight fecal samples from four white tailed deer were collected. Four samples are from animal \#1553 and \#1548 after 6 and 18 months of inoculation, and another set of four samples received from \#1542 and \#1555 after 6 and 30 months of inoculation were used to run the assay. All fecal samples were NaPTA enriched and tested for prion detection in RT-QuIC, and assays were measured at $37^{\circ} \mathrm{C}$ in the presence of $500 \mathrm{mM} \mathrm{NaCl}$ (Figure 5). However, most assays did not show any increase of ThT fluorescence except the one seeded with \#1542 (30 months post inoculation). With low detection under these conditions, assays were repeated at higher temperatures (42 or $48^{\circ} \mathrm{C}$ ) using both $400 \mathrm{mM}$ and $500 \mathrm{mM} \mathrm{NaCl}$ (Figure 6).
This increased overall conversion as evidenced by increased ThT fluorescence (Figure 6). Assays run at $42^{\circ} \mathrm{C}$ in the presence of $500 \mathrm{mM} \mathrm{NaCl}$ showed the increase of ThT fluorescence within $60 \mathrm{~h}$ of lag time for most fecal sample collected from biopsy, and the reactions were well-separated from the assay seeded with feces of negative control animal (Figure 6B). Assays running at $48^{\circ} \mathrm{C}$ not only shorten the lag time by $\sim 20 \mathrm{~h}$ for most fecal samples but it also shortens the lag time for negative control samples preventing the discrimination of positive samples from negative sample. Figure 7 shows the lag time for all seeding activity with a cutoff line for time to positive threshold such that positive samples are below the line. In terms of specificity, reactions under $42^{\circ} \mathrm{C}$ with $500 \mathrm{mM} \mathrm{NaCl}$ allowed the best results, and increasing temperature to $48^{\circ} \mathrm{C}$ improved the conversion efficiency but due to conversion in the negative control samples, the ability to discriminate positive from negative samples was not sufficient for use. It is worth noting that ThT fluorescence intensity from assays seeded with negative samples (Figure 6) is lower relative to the assays seeded with positive samples. For example, assays run at $48^{\circ} \mathrm{C}$ with $500 \mathrm{mM} \mathrm{NaCl}$ show higher ThT fluorescence intensity for positive samples compared to the intensity for negative samples.

\section{Sodium lodide Reduces Lag Time in the Detection of CWD Prions in Fecal Samples Collected From White-Tailed Deer in the Preclinical Stage of Prion Disease}

We also tested another salt, $\mathrm{NaI}$, in the reaction mixtures for detecting prions from fecal samples that were collected at times of routine animal handling. Instead of using $\mathrm{NaCl}$, 400 or $500 \mathrm{mM}$ of $\mathrm{NaI}$ was added in the RT-QuIC reaction 
A

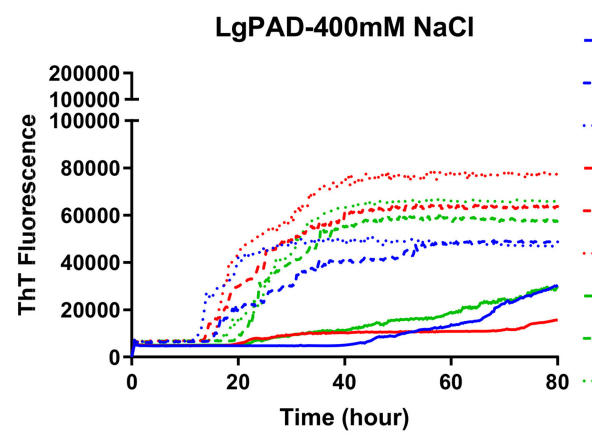

C

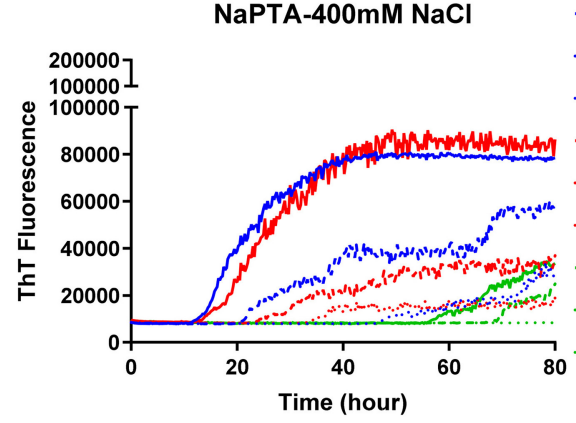

E

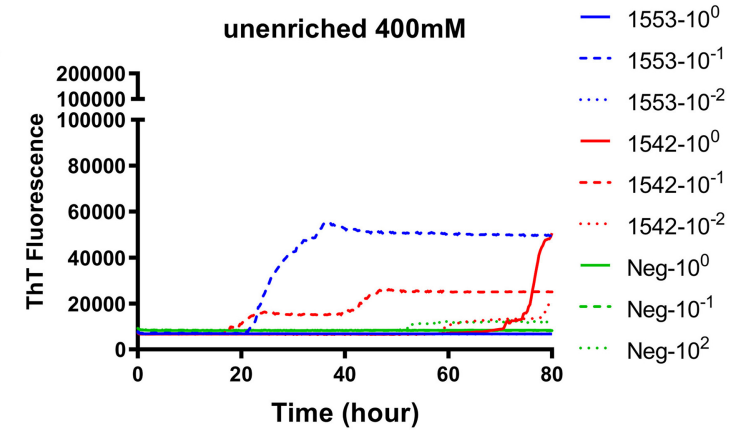

B

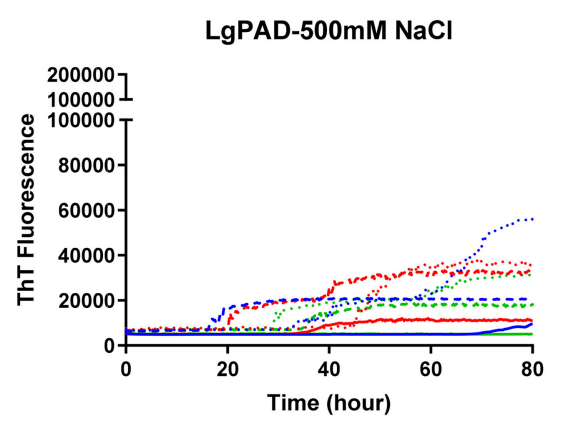

D

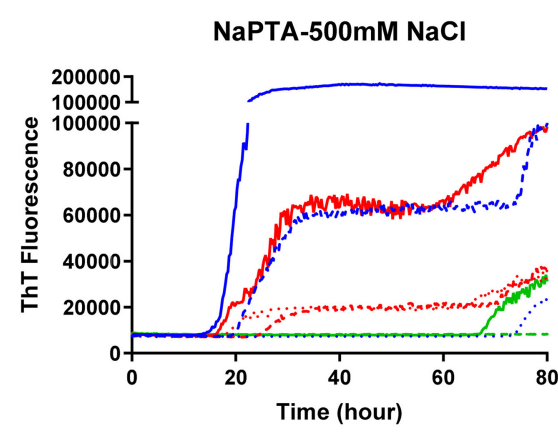

$\mathbf{F}$

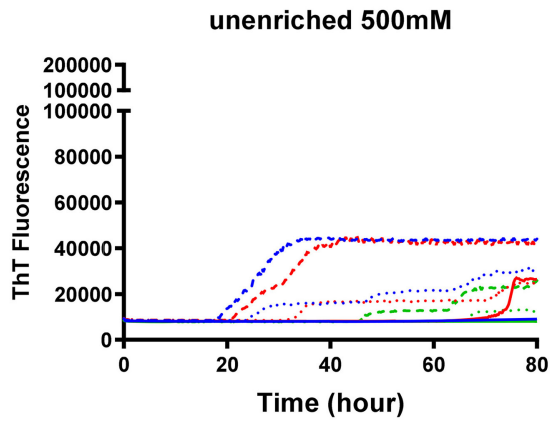

$-1553-10^{0}$

-.. $1553-10^{-1}$

‥ $1553-10^{-2}$

$-1542-10^{0}$

-.. $1542-10^{-1}$

… $1542-10^{-2}$

- Neg- $10^{0}$

-.. Neg-10-1

... Neg- $10^{-2}$

$-1553-10^{0}$

-. $1553-10^{-1}$

… $1553-10^{-2}$

- $1542-10^{0}$

-. $1542-10^{-1}$

‥ $1542-10^{-2}$

- Neg- $10^{0}$

-.. Neg- $10^{-1}$

Neg-10 ${ }^{-2}$

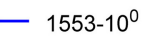

-.. $1553-10^{-1}$

‥ $1553-10^{-2}$

$-1542-10^{0}$

-.. $1542-10^{-1}$

... $1542-10^{-2}$

- Neg- $10^{0}$

-.. Neg-10-1

. Neg- $10^{2}$

FIGURE 4 | Comparison of RT-QulC reactions seeded with fecal dilutions from different enrichment. (A,B) enriched with PAD-Beads large scale (C,D) enriched with NaPTA (E,F) non-enriched dilutions. All reactions were seeded with fecal homogenate of white-tailed deer with the addition of $0.001 \%$ of SDS. Shown are the average ThT fluorescence readings determined from all replicates (four replicate reactions per each dilution).

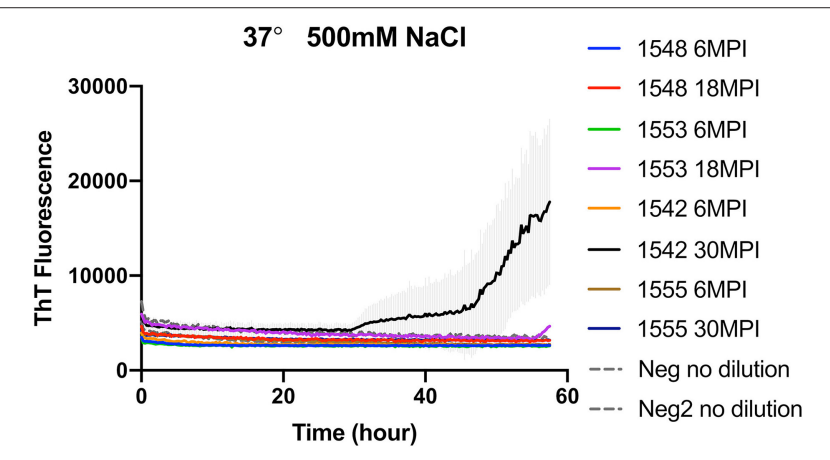

FIGURE 5 | RT-QulC reactions seeded with biopsy fecal homogenates from preclinical white- tailed deer. RT-QulC reactions were seeded with $10^{-1}$ dilution of $10 \%$ fecal homogenate. All reaction mixtures contain $500 \mathrm{mM} \mathrm{NaCl}$ and measured at $37^{\circ} \mathrm{C}$. Shown are the average ThT fluorescence readings determined from all replicates (four replicate reactions per each dilution). mixtures and they were measured either at $42^{\circ} \mathrm{C}$ or $48^{\circ} \mathrm{C}$. Overall, most reaction assays containing $\mathrm{NaI}$ showed a shorter lag time compared to the assays containing $\mathrm{NaCl}$ (Figure 8). Again, reactions performed at $48^{\circ} \mathrm{C}$ shorten lag time with $\mathrm{NaI}$ but the high temperature stimulated spontaneous reactions for negative control in early lag time. Among the reaction conditions, assays containing $500 \mathrm{mM} \mathrm{NaCl}$ measured at $42^{\circ} \mathrm{C}$ were chosen for optimal conditions to detect prions from biopsy fecal samples when considering both conversion efficiency and specificity. Figure 9 shows comparison between assays run with $500 \mathrm{mM}$ $\mathrm{NaCl}$ or $500 \mathrm{mM} \mathrm{NaI}$. In addition, Table 2 shows the lag time analysis for the comparison of all the assays containing $\mathrm{NaCl}$ and $\mathrm{NaI}$ in different temperatures and concentrations. Overall, the replacement of $\mathrm{NaCl}$ with $\mathrm{NaI}$ allowed us to detect $\mathrm{PrP}^{\mathrm{Sc}}$ from fecal samples in short lag time but with a reduced separation from the negative control. This is most apparent in Figure 9 where two inoculated animals at six MPI were no longer differentiated 
A

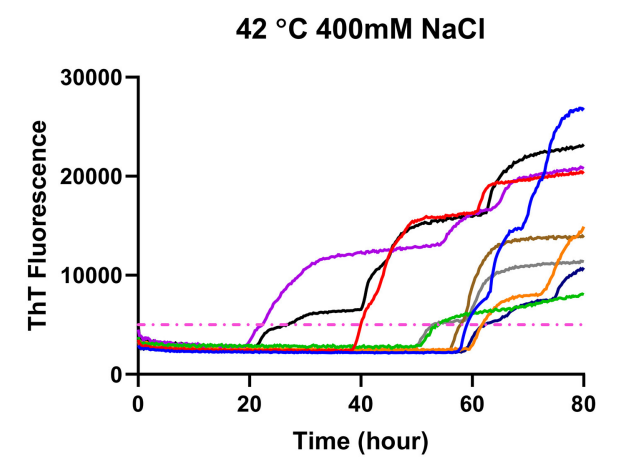

C

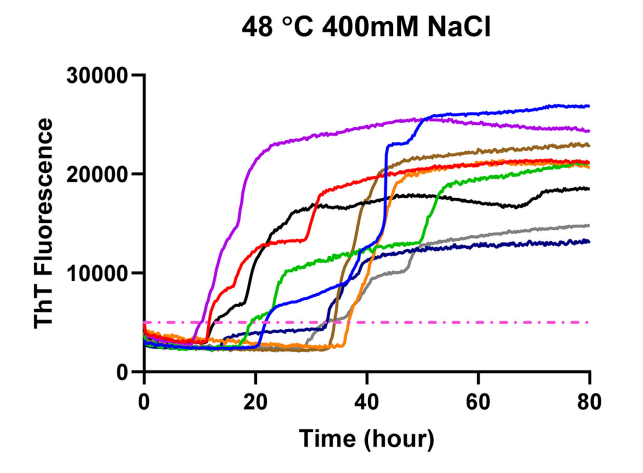

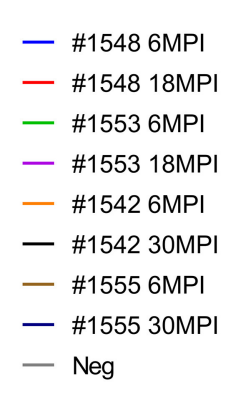

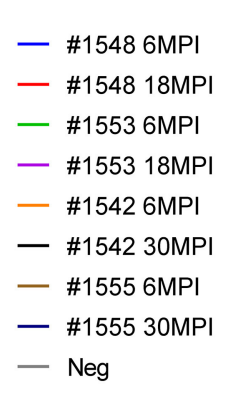

B

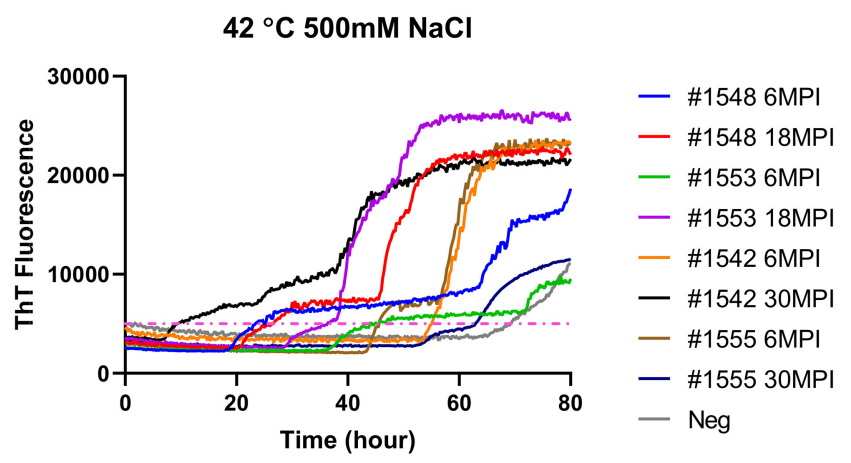

D

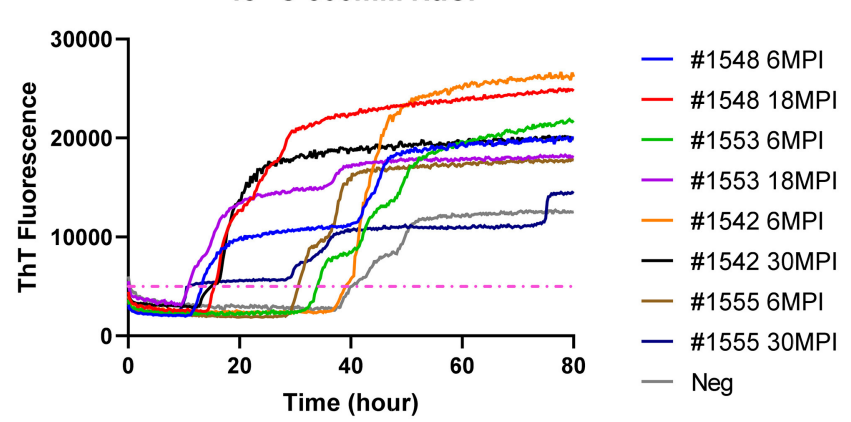

FIGURE 6 | RT-QuIC reactions seeded with biopsy fecal homogenates from preclinical white- tailed deer. Reactions conditions for each sample are followed as (A) $400 \mathrm{mM} \mathrm{NaCl}$ at $42^{\circ} \mathrm{C}$, (B) $500 \mathrm{mM}$ at $42^{\circ} \mathrm{C}$, (C) $400 \mathrm{mM}$ at $48^{\circ} \mathrm{C}$, and (D) $500 \mathrm{mM}$ at $48^{\circ} \mathrm{C}$. Shown are the average ThT fluorescence readings determined from all replicates (four replicate reactions per each dilution).

from negative. This experiment indicates that $\mathrm{NaCl}$ is still the better salt choice for fecal samples using bank vole substrate since all fecal samples from inoculated animals exhibited ThT fluorescence indicative of fibril formation in a shorter time relative to the negative control samples.

\section{DISCUSSION}

In this study, we have optimized RT-QuIC reaction conditions to enhance the detection of $\mathrm{PrP}^{\mathrm{Sc}}$ from fecal homogenates from clinical and preclinical white-tailed deer. Different factors including salt concentrations and temperature were tested with regard to sensitivity and specificity of detection of infectious prions from fecal samples using bank vole recombinant prion protein as the amplification substrate. For fecal samples collected from clinically affected deer, a reaction temperature of $37^{\circ} \mathrm{C}$ showed prion detection, as previously reported by Henderson and colleagues where they observed less spontaneous reactions by reducing reaction temperature from 42 to $37^{\circ} \mathrm{C}$ for fecal prion detection in RT-QuIC (8). In the data presented in this study, the assays seeded with fecal samples from clinically affected animals showed prion seeding activity at $37^{\circ} \mathrm{C}$ even without any enrichment. However, for fecal samples collected from preclinical deer inoculated with CWD, RT-QuIC conditions had to be further optimized to enhance the prion detection. Using high salt concentration like $500 \mathrm{mM} \mathrm{NaCl}$ and increasing temperature from 37 to $42^{\circ} \mathrm{C}$ enhanced the seeding activity of fecal samples and allowed for discrimination between assays seeded with positive fecal samples and assays seeded with negative controls. We also tested higher temperatures like $48^{\circ} \mathrm{C}$ for better sensitivity of fecal prions in RT-QuIC. Higher temperatures did shorten the lag time of seeding activity overall, however, assays performed at $48^{\circ} \mathrm{C}$ also shorten the lag time of assays seeded with negative control, which led to an inability to discriminate positive samples from negative samples. Modified conditions were identified that enhance detection of prions in fecal samples from preclinical animals. Further, it was ultimately determined that for RT-QuIC reactions seeded with fecal samples from preclinical animals, it is desirable to incorporate NaPTA enrichment prior to RT-QuIC for improved detection of seeding activity.

A previously published report that had applied different ions of the Hofmeister series of ions to RT-QuIC reactions showed enhanced sensitivity of seeding activity for assays seeded with ear homogenate from CWD infected deer in the presence of $\mathrm{NaI}$ instead of $\mathrm{NaCl}$ (28). Here, we compare the seeding activity of fecal samples in the presence of $\mathrm{NaI}$ to that of $\mathrm{NaCl}$. As can be seen in Figure 8, similar result was observed for fecal samples as was previously reported for ear homogenate such that assays containing $\mathrm{NaI}$ had shorter lag times than assays containing $\mathrm{NaCl}$. Assays seeded with negative sample 

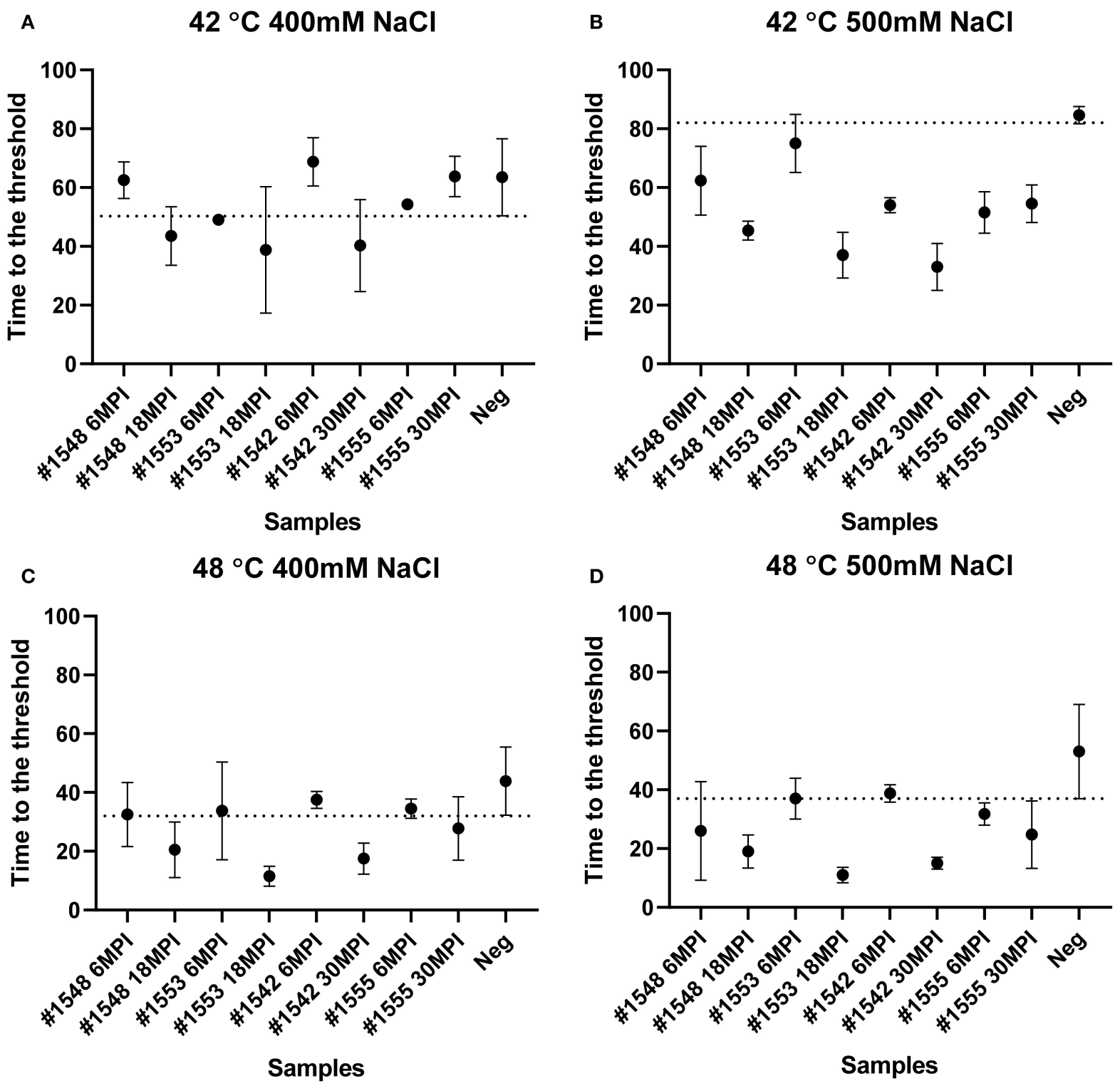

FIGURE 7 | Lag time analysis of RT-QuIC reactions seeded with biopsy fecal homogenates from preclinical white-tailed deer. Reaction conditions for each sample are followed as (A) $400 \mathrm{mM} \mathrm{NaCl}$ at $42^{\circ} \mathrm{C}$, (B) $500 \mathrm{mM} \mathrm{NaCl}$ at $42^{\circ} \mathrm{C}$, (C) $400 \mathrm{mM} \mathrm{NaCl}$ at $48^{\circ} \mathrm{C}$, and (D) $500 \mathrm{mM} \mathrm{NaCl}$ at $48^{\circ} \mathrm{C}$. Circles represent the mean and bars represent SD of four replicate reactions. The horizontal line is the cutoff.

also showed a shortened lag time for unseeded fibril formation under all reaction temperatures complicating discrimination of positive and negative samples. When we compare the results of RT-QuIC with $\mathrm{NaI}$ and $\mathrm{NaCl}$, it is clear that $\mathrm{NaI}$ could be useful for quick detection with samples that have relatively high amount of prions. However, $\mathrm{NaCl}$, despite the longer lag time, provided $100 \%$ sensitivity with better separation of positive assays from negative assays. This is in contrast to the previous report utilizing $\mathrm{NaI}$ where assays seeded with ear homogenate from CWD infected deer in the presence of NaI showed higher sensitivity and better specificity for prion detection than $\mathrm{NaCl}$ when used in RT-QuIC assays (28).
It is well-documented that fecal samples from cervids infected with CWD contain detectable prions (8-11). RT-QuIC seems practical to detect infectious prions from fecal samples of CWD infected cervids based on our study and previous reports. John and colleagues published a report that had shown RT-QuIC detection of fecal samples obtained from preclinical white-tailed deer. They tested fecal samples collected 20 and 30 months post inoculation, and they were only able to detect $\mathrm{PrP}^{\mathrm{Sc}}$ from fecal sample collected at 30 months post inoculation possibly due to non-enrichment of the samples (11). Later, Cheng and colleagues showed that fecal prions of CWD infected elk could be detected with a NaPTA enrichment and detection by RT-QuIC 
A

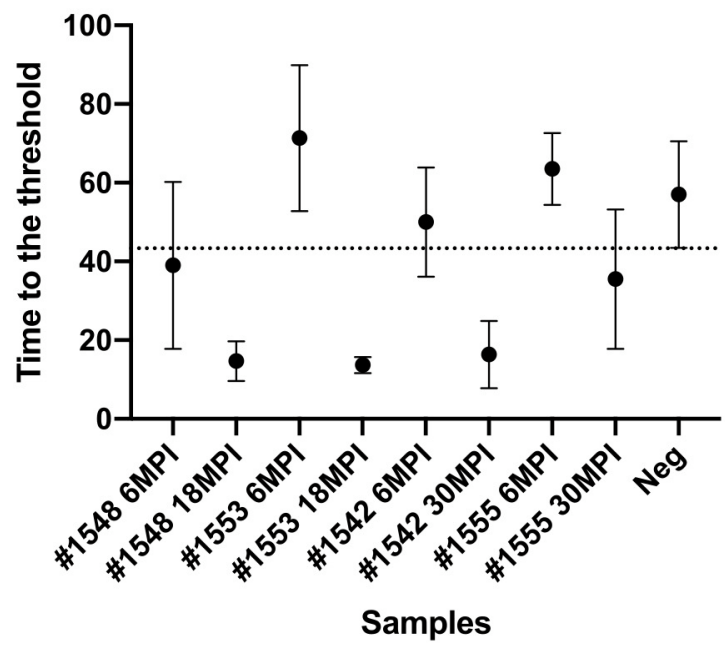

C

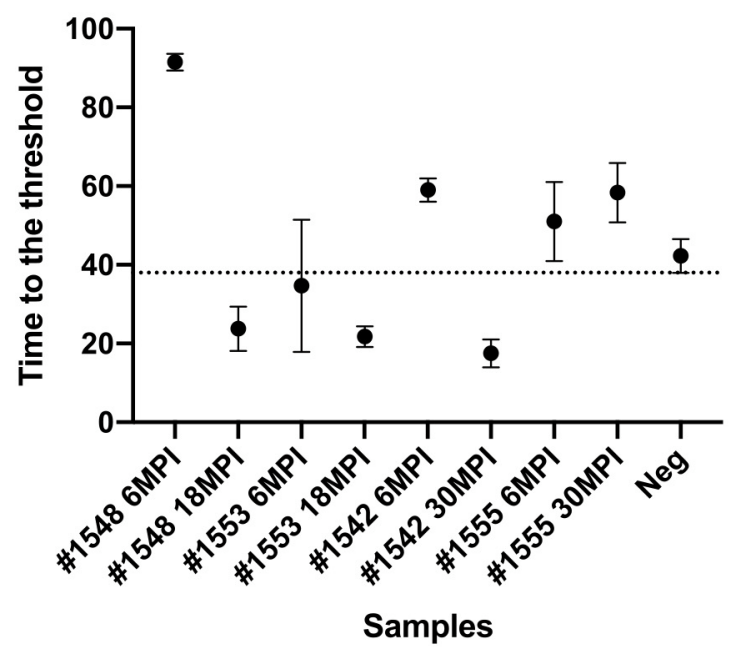

B

$42{ }^{\circ} \mathrm{C} 500 \mathrm{mM}$ Nal

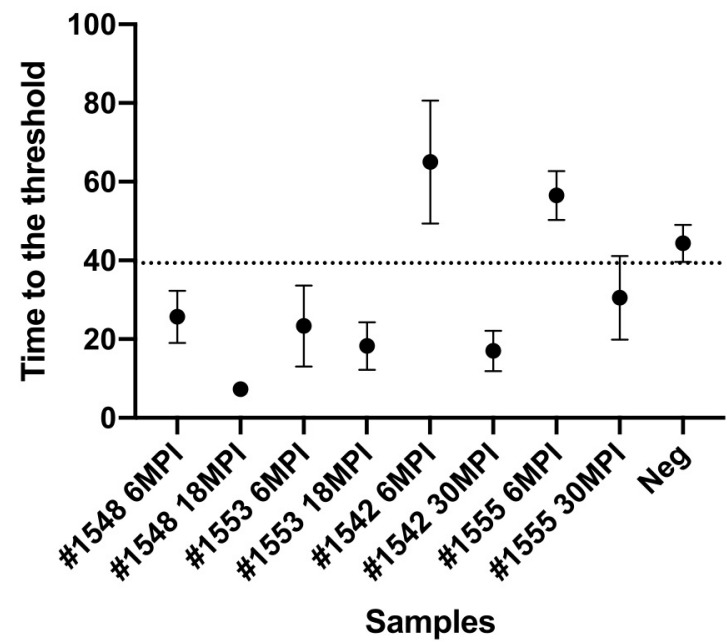

D
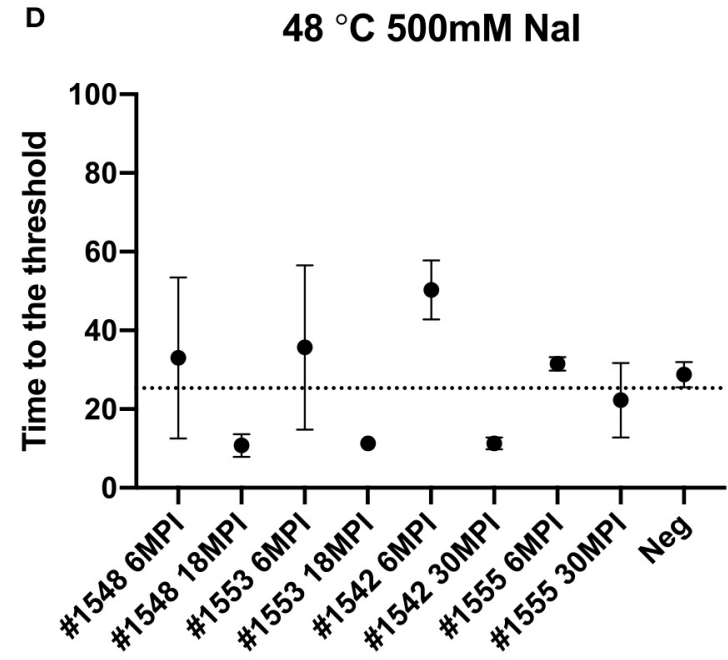

Samples

FIGURE 8 | Lag time analysis of RT-QulC reactions seeded with biopsy fecal homogenates from preclinical white-tailed deer in the presence of Nal. Reactions conditions for each sample are followed as (A) $400 \mathrm{mM} \mathrm{Nal}$ at $42^{\circ} \mathrm{C}$, (B) $500 \mathrm{mM} \mathrm{Nal}$ at $42^{\circ} \mathrm{C}$, (C) $400 \mathrm{mM} \mathrm{Nal} \mathrm{at} 48^{\circ} \mathrm{C}$, and (D) $500 \mathrm{mM} \mathrm{Nal}$ at $48{ }^{\circ} \mathrm{C}$. Circles represent the mean and bars represent SD of four replicate reactions. The horizontal line is the cutoff.

using recombinant mouse prion protein (10). Given their choice of substrate and reaction condition, they found it necessary to replace the substrate after $24 \mathrm{~h}$ of $\mathrm{RT}$-QuIC reaction in order to see better detection of fecal prions. Henderson and colleagues also published a report indicating that fecal prions could be detected by RT-QuIC using Syrian hamster recombinant prion protein substrate and iron-oxide bead extraction as an enrichment rather than NaPTA precipitation (8). They also evaluated the reaction temperature, 37,40 , or $42^{\circ} \mathrm{C}$ to reduce the lag time for detection of positive samples and decrease spontaneous fibril formation in negative controls finding $37^{\circ} \mathrm{C}$ to be optimal for preventing spontaneous reactions while still allowing good sensitivity. In this work, we used recombinant
BV substrate which is generally reported as a universal substrate for detecting various prion diseases from both animals and humans by RT-QuIC (19) to detect CWD prions in fecal samples from white-tailed deer intranasal inoculated with CWD sourced from experimentally passaged through either white-tailed deer or racoon. Like Cheng and colleagues, we applied NaPTA precipitation for enrichment of prions in all fecal samples we collected. We also applied another enrichment methodology PAD-Beads, and has been previously reported for successfully enhanced RT-QuIC assays with brain homogenate of TSE inoculated animals (16). However, when PAD-Beads enrichment was applied for fecal samples, reactions with negative fecal samples were also seeded, which suggested that PAD-Beads may 

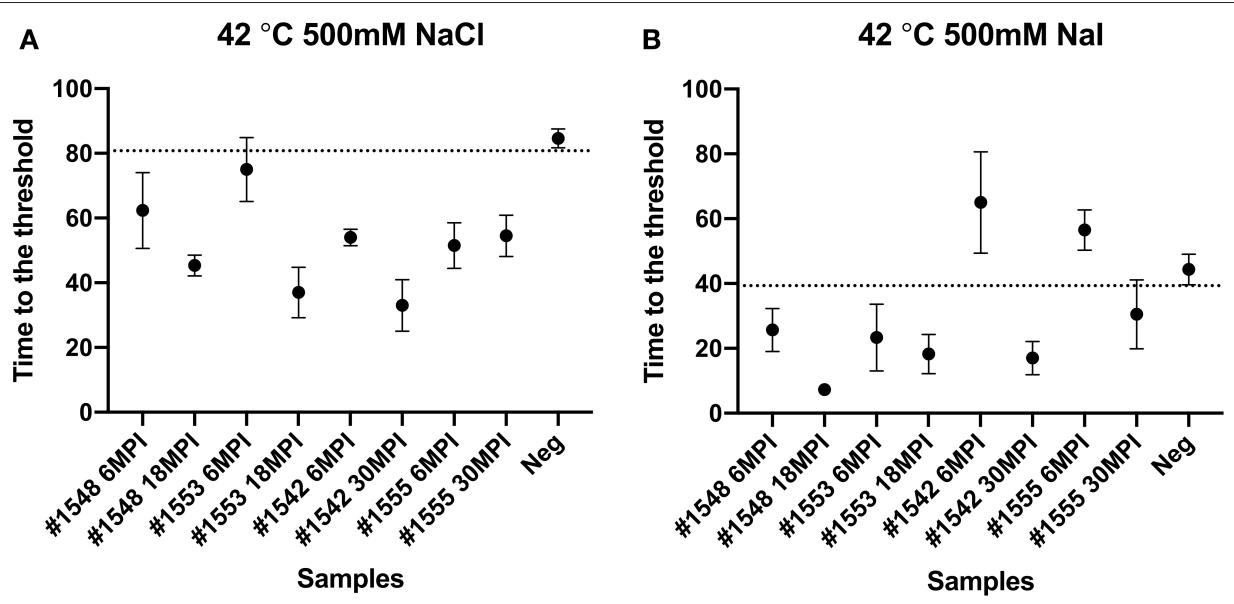

FIGURE 9 | Comparison of lag time analysis of RT-QulC reactions seeded with biopsy fecal homogenates from preclinical white-tailed deer in the presence of $500 \mathrm{mM}$ $\mathrm{NaCl}$ (A) or $\mathrm{Nal}$ (B). Circles represent the mean and the bars represent SD of four replicate reactions. The horizontal line is the cutoff.

TABLE 2 | Lag time analysis from real-time quacking-induced conversion reactions seeded with preclinical fecal samples in different temperatures, different salt types, and concentrations.

\begin{tabular}{llllllllll}
\hline & \#1548 6 MPI & \#1548 $\mathbf{1 8}$ MPI & \#1553 6 MPI & \#1553 18 MPI & \#1542 6 MPI & \#1542 $\mathbf{3 0}$ MPI & \#1555 6 MPI & \#1555 30 MPI & Neg \\
\hline $42^{\circ} \mathrm{C} 400 \mathrm{mM} \mathrm{Nal}$ & $39.0 \pm 21.2$ & $14.7 \pm 5.0$ & $71.3 \pm 18.5$ & $13.7 \pm 2.0$ & $50.0 \pm 13.8$ & $16.3 \pm 8.5$ & $63.5 \pm 9.1$ & $35.5 \pm 17.6$ & $57.0 \pm 13.6$ \\
$42^{\circ} \mathrm{C} 500 \mathrm{mM} \mathrm{Nal}$ & $25.7 \pm 6.7$ & $7.25 \pm 1.9$ & $23.3 \pm 10.3$ & $18.3 \pm 6.0$ & $65.0 \pm 15.6$ & $17.0 \pm 5.1$ & $56.5 \pm 6.2$ & $30.5 \pm 10.6$ & $44.3 \pm 4.7$ \\
$48^{\circ} \mathrm{C} 400 \mathrm{mM} \mathrm{Nal}$ & $91.5 \pm 2.1$ & $23.8 \pm 5.6$ & $34.7 \pm 16.8$ & $21.8 \pm 2.6$ & $59.0 \pm 2.9$ & $17.5 \pm 3.5$ & $51.0 \pm 10.0$ & $58.3 \pm 7.6$ & $42.3 \pm 4.3$ \\
$48^{\circ} \mathrm{C} 500 \mathrm{mM} \mathrm{Nal}$ & $33.0 \pm 20.0$ & $10.8 \pm 2.9$ & $35.7 \pm 20.8$ & $11.3 \pm 1.0$ & $50.3 \pm 7.5$ & $11.3 \pm 1.5$ & $31.5 \pm 1.7$ & $22.3 \pm 9.5$ & $28.8 \pm 3.2$ \\
$42^{\circ} \mathrm{C} 400 \mathrm{mM} \mathrm{NaCl}$ & $62.5 \pm 6.2$ & $43.5 \pm 9.9$ & $49 \pm 1.6$ & $38.7 \pm 21.5$ & $68.7 \pm 8.2$ & $40.3 \pm 15.6$ & $54.3 \pm 1.5$ & $63.7 \pm 6.9$ & $63.5 \pm 13.1$ \\
$42^{\circ} \mathrm{C} 500 \mathrm{mM} \mathrm{NaCl}$ & $62.3 \pm 11.7$ & $45.3 \pm 3.2$ & $75.0 \pm 9.9$ & $37.0 \pm 7.8$ & $54.0 \pm 2.6$ & $33.0 \pm 7.9$ & $51.5 \pm 7.0$ & $54.5 \pm 6.4$ & $84.6 \pm 2.9$ \\
$48^{\circ} \mathrm{C} 400 \mathrm{mM} \mathrm{NaCl}$ & $32.5 \pm 10.9$ & $20.5 \pm 9.5$ & $33.8 \pm 16.6$ & $11.5 \pm 3.4$ & $37.5 \pm 2.9$ & $17.5 \pm 5.3$ & $34.5 \pm 3.3$ & $27.8 \pm 10.8$ & $43.9 \pm 11.6$ \\
$48^{\circ} \mathrm{C} 500 \mathrm{mM} \mathrm{NaCl}$ & $26.0 \pm 16.7$ & $19.0 \pm 5.6$ & $37.0 \pm 6.9$ & $11.0 \pm 2.6$ & $38.7 \pm 2.9$ & $15.0 \pm 2.0$ & $31.7 \pm 3.7$ & $24.7 \pm 11.4$ & $53.0 \pm 16.1$
\end{tabular}

All times were recorded in hours.

not be suitable for fecal sample enrichment coupled with RTQuIC. Most notable in this work is that by 6 months postinoculation, prion seeding is identified in both TSE isolates. For the samples included in this study, white-tailed deer CWD showed an incubation time in the 21-24 months range, while CWD passaged through raccoons prior to inoculation in whitetailed deer exhibited an onset of clinical signs in excess of 34 months with one of our samples not showing clinical signs at 56 months post inoculation. This highlights the potential for early clinical detection of TSEs using NaPTA enrichment coupled with RT-QuIC for the detection of CWD. Overall, combination of BV substrate, different salt concentration, and temperature allowed us to detect infectious prions within short lag time with good specificity from clinical or preclinical white-tailed deer.

Altogether, we confirm again that RT-QuIC is a powerful tool to detect infectious fecal prions from CWD infected white-tailed deer. Use of feces is a non-invasive and non-stressing approach to sampling of animals, of particular importance for nondomesticated animals that may be less tolerant to the handling required for sampling by other means. This is of importance to the management of both wild and farmed cervids and is also of use in experimental settings where repeated sampling of an individual animal would be otherwise difficult. Ultimately, fecal sampling may prove useful in the determination of disease prevalence in a geographic region or within a herd.

\section{DATA AVAILABILITY STATEMENT}

The original contributions presented in the study are included in the article/supplementary material, further inquiries can be directed to the corresponding author.

\section{ETHICS STATEMENT}

The animal study was reviewed and approved by National Animal Disease Center Institutional Animal Care and Use Committee.

\section{AUTHOR CONTRIBUTIONS}

SH and EN: conceptualization, methodology, and writing original draft. SH: formal analysis and investigation. EN and JG: 
resources. EN: supervision. $\mathrm{SH}, \mathrm{EN}$, and JG: writing review and editing. All authors contributed to the article and approved the submitted version.

\section{FUNDING}

This research was funded in its entirety by congressionally appropriated funds to the United States Department of

\section{REFERENCES}

1. Haley NJ, Hoover EA. Chronic wasting disease of cervids: current knowledge and future perspectives. Annu Rev Anim Biosci. (2015) 3:30525. doi: 10.1146/annurev-animal-022114-111001

2. Benestad SL, Mitchell G, Simmons M, Ytrehus B, Vikoren T. First case of chronic wasting disease in Europe in a Norwegian free-ranging reindeer. Vet Res. (2016) 47:88. doi: 10.1186/s13567-016-0375-4

3. Department for Environment FaRA, Agency APH, VSPAT-ID. Monitoring, Update on Chronic Wasting Disease in Europe (2018). Available online at: https://assets.publishing.service.gov.uk/government/uploads/system/ uploads/attachment_data/file/703368/sa-cwd-norway-20180425.pdf

4. Swedish National Veterinary Institute. Map of Chronic Wasting Disease (CWD), Uppsala (2019).

5. Prusiner SB. Prions. Proc Natl Acad Sci USA. (1998) 95:1336383. doi: 10.1073/pnas.95.23.13363

6. Collinge J. Prion diseases of humans and animals: their causes and molecular basis. Annu Rev Neurosci. (2001) 24:51950. doi: 10.1146/annurev.neuro.24.1.519

7. Caughey B, Chesebro B. Transmissible spongiform encephalopathies and prion protein interconversions. Adv Virus Res. (2001) 56:277311. doi: 10.1016/S0065-3527(01)56031-5

8. Henderson DM, Tennant JM, Haley NJ, Denkers ND, Mathiason CK, Hoover EA. Detection of chronic wasting disease prion seeding activity in deer and elk feces by real-time quaking-induced conversion. J Gen Virol. (2017) 98:1953-62. doi: 10.1099/jgv.0.000844

9. Cheng YC, Hannaoui S, John TR, Dudas S, Czub S, Gilch S. Real-time quaking-induced conversion assay for detection of CWD prions in fecal material. J Vis Exp. (2017) 127:56373. doi: 10.3791/56373

10. Cheng YC, Hannaoui S, John TR, Dudas S, Czub S, Gilch Early S. Non-invasive detection of chronic wasting disease prions in elk feces by real-time quaking induced conversion. PLoS ONE. (2016) 11:e0166187. doi: 10.1371/journal.pone.0166187

11. John TR, Schatzl HM, Gilch S. Early detection of chronic wasting disease prions in urine of pre-symptomatic deer by real-time quaking-induced conversion assay. Prion. (2013) 7:253-8. doi: 10.4161/pri.24430

12. Henderson DM, Denkers ND, Hoover CE, Garbino N, Mathiason CK, Hoover EA. Longitudinal detection of prion shedding in saliva and urine by chronic wasting disease-infected deer by real-time quaking-induced conversion. $J$ Virol. (2015) 89:9338-47. doi: 10.1128/JVI.01118-15

13. Elder AM, Henderson DM, Nalls AV, Wilham JM, Caughey BW, Hoover EA, et al. In vitro detection of prionemia in TSE-infected cervids and hamsters. PLoS ONE. (2013) 8:e80203. doi: 10.1371/journal.pone.0080203

14. Atarashi R, Sano K, Satoh K, Nishida N. Real-time quaking-induced conversion: a highly sensitive assay for prion detection. Prion. (2011) 5:1503. doi: 10.4161/pri.5.3.16893

15. Moore SJ, Smith JD, Greenlee MH, Nicholson EM, Richt JA, Greenlee JJ. Comparison of two US sheep scrapie isolates supports identification as separate strains. Vet Pathol. (2016) 53:1187-96. doi: 10.1177/0300985816629712

16. Hwang S, Dassanayake RP, Nicholson EM. PAD-beads enrichment enhances detection of $\operatorname{PrP}(\mathrm{Sc})$ using real-time quaking-induced conversion. BMC Res Notes. (2019) 12:806. doi: 10.1186/s13104-0194842-7

17. Vrentas CE, Onstot S, Nicholson EM. A comparative analysis of rapid methods for purification and refolding of recombinant bovine prion protein. Protein Expr Purif. (2012) 82:380-8. doi: 10.1016/j.pep.2012. 02.008
Agriculture, Agriculture Research Service. The funder of the work did not influence study design, data collection and analysis, decision to publish, and preparation of the manuscript.

\section{ACKNOWLEDGMENTS}

The authors thank Semakaleng Lebepe-Mazur and Joseph Lesan for providing technical support for this project.

18. Hwang S, Tatum T, Lebepe-Mazur S, Nicholson EM. Preparation of lyophilized recombinant prion protein for TSE diagnosis by RT-QuIC. BMC Res Notes. (2018) 11:895. doi: 10.1186/s13104-018-3982-5

19. Orru CD, Groveman BR, Raymond LD, Hughson AG, Nonno R, Zou W, et al. Bank Vole prion protein as an apparently universal substrate for RT-QuICbased detection and discrimination of prion strains. PLoS Pathog. (2015) 11:e1004983. doi: 10.1371/journal.ppat.1004983

20. Dassanayake RP, Orru CD, Hughson AG, Caughey B, Graca T, Zhuang $\mathrm{D}$, et al. Sensitive and specific detection of classical scrapie prions in the brains of goats by real-time quaking-induced conversion. J Gen Virol. (2016) 97:803-12. doi: 10.1099/jgv.0.000367

21. Cheng K, Sloan A, Avery KM, Coulthart M, Carpenter M, Knox JD. Exploring physical and chemical factors influencing the properties of recombinant prion protein and the real-time quaking-induced conversion (RT-QuIC) assay. PLoS ONE. (2014) 9:e84812. doi: 10.1371/journal.pone.0084812

22. Orru CD, Hughson AG, Groveman BR, Campbell KJ, Anson KJ, Manca $\mathrm{M}$, et al. Factors that improve RT-QuIC detection of prion seeding activity. Viruses. (2016) 8:140. doi: 10.3390/v8050140

23. Masujin K, Orru CD, Miyazawa K, Groveman BR, Raymond LD, Hughson AG, et al. Detection of atypical H-type bovine spongiform encephalopathy and discrimination of bovine prion strains by real-time quaking-induced conversion. J Clin Microbiol. (2016) 54:676-86. doi: 10.1128/JCM.02731-15

24. Orru CD, Favole A, Corona C, Mazza M, Manca M, Groveman BR, et al. Detection and discrimination of classical and atypical L-type bovine spongiform encephalopathy by real-time quaking-induced conversion. J Clin Microbiol. (2015) 53:1115-20. doi: 10.1128/JCM.02906-14

25. Hwang S, Greenlee JJ, Nicholson EM. Use of bovine recombinant prion protein and real-time quaking-induced conversion to detect cattle transmissible mink encephalopathy prions and discriminate classical and atypical L- and H-Type bovine spongiform encephalopathy. PLoS ONE. (2017) 12:e0172391. doi: 10.1371/journal.pone.0172391

26. Orru CD, Groveman BR, Hughson AG, Zanusso G, Coulthart MB, Caughey B. Rapid sensitive RT-QuIC detection of human Creutzfeldt-Jakob disease using cerebrospinal fluid. MBio. (2015) 6:e02451-14. doi: 10.1128/mBio.02451-14

27. Orru CD, Bongianni M, Tonoli G, Ferrari S, Hughson AG, Groveman BR, et al. A test for Creutzfeldt-Jakob disease using nasal brushings. N Engl J Med. (2014) 371:519-29. doi: 10.1056/NEJMoa1315200

28. Metrick MA II, do Carmo Ferreira N, Saijo E, Hughson AG, Kraus A, Orru C, et al. Million-fold sensitivity enhancement in proteopathic seed amplification assays for biospecimens by Hofmeister ion comparisons. Proc Natl Acad Sci USA. (2019) 116:23029-39. doi: 10.1073/pnas.1909322116

Disclaimer: Mention of trade names or commercial products in this publication is solely for the purpose of providing specific information and does not imply recommendation or endorsement by the United States Department of Agriculture (USDA). USDA is an equal opportunity provider and employer.

Conflict of Interest: The authors declare that the research was conducted in the absence of any commercial or financial relationships that could be construed as a potential conflict of interest.

Copyright (C) 2021 Hwang, Greenlee and Nicholson. This is an open-access article distributed under the terms of the Creative Commons Attribution License (CC BY). The use, distribution or reproduction in other forums is permitted, provided the original author(s) and the copyright owner(s) are credited and that the original publication in this journal is cited, in accordance with accepted academic practice. No use, distribution or reproduction is permitted which does not comply with these terms. 